DOI: https://doi.org/10.33103/uot.ijccce.20.4.1

\title{
The Design and Simulation of FBG Sensors for Medical Application
}

\author{
Mohamed J. Ali ${ }^{1}$, Alaa H. Ali ${ }^{2}$, Aseel I. Mahmood ${ }^{3}$, Mohammed A hussien ${ }^{4}$ \\ 1,2,3,4 Department of Electrical Engineering, University of Technology,,Bagdad, Iraq \\ mohamedjamal964@gmail.com,alaa_eng1970@yahoo.com, aseelibrahim208@gmail.com, \\ 14003@uotechnology.edu.iq
}

\begin{abstract}
Fiber Bragg Grating sensors have a wide range of applications, ranging from their use for health monitoring, in medical applications and also as biomedical sensors, among others. Moreover, since fiber Bragg gratings have many advantages that qualify them to be of great benefit, of course, the most important of these applications are vital signs of human health condition Such as blood pressure, heart rate (pulse rate), and body temperature. The Vital-signs are noticeable variables. The temperature and blood pressure are changed according to the physical, involuntary, nervous and psychological state of the person. Therefore, the measurement of vital signs is very necessary, In this work, fiber Bragg grating sensor has been designed and simulated to study the performance of fiber Bragg grating sensor as a Body temperature of human beings ranged from $\left(35^{\circ} \mathrm{C}\right.$ to $40^{\circ} \mathrm{C}$, which is from hypothermia to hyperthermia) and blood pressure that ranged between lower and higher extremities (40 to $190 \mathrm{mmHg}$ ) from hypotension to hypertension, using optigrating and optisystem simulation softwar. The designed sensor was very sensitive to human temperature and blood pressure ranges which were $13.632 \mathrm{pm} / \mathrm{oC}$ and 15.75 $\mathrm{pm} / \mathrm{mm} \mathrm{Hg}$, respectively.
\end{abstract}

Index Terms - fiber Bragg grating, fiber optic temperature sensor, optical wavelength, modulation, high accuracy sensors, health monitoring system.

\section{INTRODUCTION}

The development of optical fiber communication technology has contributed to the development and intensification of studies of optical fiber sensors [1] more suitable and maybe more precisely for sensing [2], The sensors based on optical fiber other than electronic sensors possess several important advantages, including low manufacturing cost [3], A Fiber Bragg Grating ( $\mathrm{FBG}$ ) is an optical device obtained through introducing a modulation of the refractive index of the fiber core [4]. Fiber Bragg grating (FBG) has wide attracted attention because of many of their inherent advantages Such as cylindrical geometry, small size, light weight, environmentally robust compact size, high sensitivity, remote sensing capability[5,6], electromagnetic interference immunity, and radio frequency interface[7]. Win is the special attraction used for monitoring and measuring of various parameters, such as temperature [8], strain [9], bend[10], pressure[11], chemical measurement [12], humidity [13], and others. Strain and temperature measurements are of great importance to smart systems for different applications, such as patient monitoring systems [14]. The FBGs are a kind of distributed Bragg reflectors which reflects a special wavelength and transmits the others, this concept is obtained through changing the refractive index as a periodic variation of the core in optical fiber, which creates a dielectric mirror for reflecting a specific wavelength, therefore the FBG could be implemented to block the particular wavelength [15], as shown in Figure 1. 


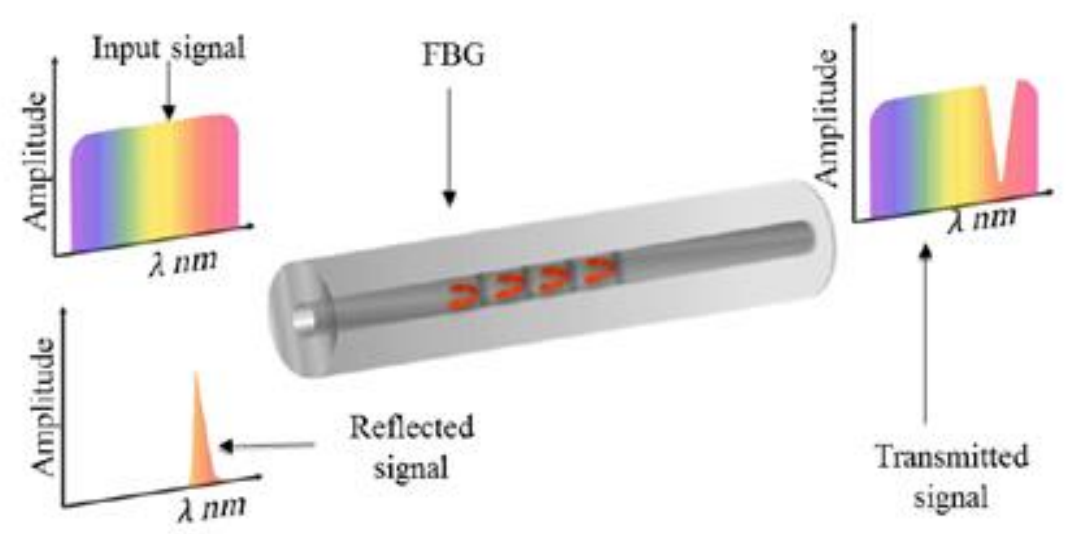

FIG. 1. THE INTERNAL STRUCTURE AND WORKING PRINCIPLE OF FIBER BRAGG GRATING [11]

The Bragg wavelength $\left(\lambda_{B}\right)$ shifting reflected from the grating is the principle of monitoring the operation of The FBG, as a function of the monitored parameter by the relation no.(1) the grating period $(\Lambda)$ and the effective refractive index of the fiber core $\left(\mathrm{n}_{\text {eff }}\right)$ are effective on The Bragg wavelength [16]:

$$
\lambda_{\mathrm{B}}=2 \mathrm{n}_{\mathrm{eff}} \Lambda
$$

Therefore, The factors affecting the change of wavelength of the Bragg are two: the effective refractive index of the optical fiber core or cthe hanges in the grain period. So, the strain and temperature, which have effect on the FBG wavelength, can be translated by [16]:

$$
\Delta \lambda_{\mathrm{B}}=\lambda_{\mathrm{B}}\left(1-\rho_{\alpha}\right) \Delta \varepsilon+\lambda_{\mathrm{B}}(\alpha+\xi)
$$

where the strain is the effect on the first term on the $\lambda_{B}$ but the temperature effect on the second term. Hence, in Eq. (2), $\Delta \lambda_{B}$ represents the shift of the Bragg wavelength, while $\xi$ thermal expansion, $\alpha$ is thermo-optic coefficients, and $\rho$ is the photoelastic of the fiber, ( $\Delta \mathrm{T}$ and $\Delta \varepsilon$ ) change directly with temperature and pressure changes respectively.

The Bragg resonance shifts are detected by different schemes, which can be very sensitive. In such a scheme, broadband light (generated by the super-luminescent diode, edge-emitting LED, Erbium-Doped Fiber EDF super fluorescent source) is injected into the fiber and the peak wavelength of the reflected light is determined. Another scheme involves interrogating the Bragg Grating with the sensor wavelength tuned by a laser or using the sensor as a tuning element in the laser. cavity, detecting a small shift in the Bragg wavelength of an FBG sensor element, which corresponds to change of the sensing parameter is important. In a laboratory environment, this can be accomplished using a high precision optical spectrum analyzer [17].

In recent years, an excellent sensor element FBG has been considered, which is currently receiving more and more research interest. The reflected wavelength from the FBG depends on physical properties such as temperature and strain. By illuminating the FBG with broadband lights, the shift in the peak reflectivity wavelength can be used as a measure for the physical property. The FBG attracts wide attention for its wavelength based algorithms, which make the sensor self-referring with less power/conductor loss [18].

Bragg grating temperature sensing occurs mainly through the temperature effect on the refractive index and, to a lesser degree, through the coefficient of expansion. It is 
noteworthy that sensitivity to temperature can be increased or multiplied by proper bonding to other materials. While for strain its depends on changing in spacing between pitch [19].

The vital signs represent the state of human health like heart rate (HR), body temperature (BT) and respiratory rate (RR) and the like. Vital signs are remarkable changes. The temperature and Blood pressure is a resulting and dependent change on the physical status, mental state, and autonomic nervous system of the human body Therefore, measuring vital signs is very necessary and should a unrestrained and non-invasive[20]. Several research articles were used FBGs have presented results of the application of FBG in the medical field like e-health monitoring, heart rate monitoring, and vital sign measurements [21-23].

In the current study, FBG had been designed and simulated for studying its performance as a temperature and pressure sensor for human in the same time with the online record without the need to restart the device as it in the traditional equipment.

The body temperature of human beings ranges from $\left(35^{\circ} \mathrm{C}\right.$ to $\left.40^{\circ} \mathrm{C}\right)$ which is from (hypothermia to hyperthermia), and the normal human temperature is typically stated as $\left(36.5{ }^{\circ} \mathrm{C}-37.5{ }^{\circ} \mathrm{C}\right)$ [24]. The blood pressure ranges between lower and higher extremities (40 diastolic to 190 systolic $\mathrm{mmHg}$ ) and from hypotension to hypertension and the normal range is $(120 / 80 \mathrm{mmHg})$ [25].

\section{The Simulation of the Model}

The Temperature and blood pressure sensors for detecting vital signs had been designed and simulated using a specialized photonic software. A uniform FBG had been designed using OptiGrating simulation software. The Bragg wavelength was $(1.55 \mu \mathrm{m})$, the grating length was $(50 \mu \mathrm{m})$, the periodicity circa $(0.533 \mu \mathrm{m})$. The performance of the FBG sensors had been accomplished using OptiSystem (V.16). Table 1. Shows the parameters of the designed components.

TABLE 1. THE PARAMETERS OF THE DESIGNED COMPONENTS.

\begin{tabular}{cc}
\hline Component & Values \\
\hline White light source & Power $=(0.001 \mathrm{mwatt}) \quad$, peak wavelength $=(1550 \mathrm{~nm})$ \\
FBG 1 & Bragg wavelength $(1550 \mathrm{~nm})$, reflectivity $(0.99)$, bandwidth $(125 \mathrm{GHz})$ \\
FBG 2 & Bragg wavelength $(1550 \mathrm{~nm})$, reflectivity $(0.99)$, bandwidth $(125 \mathrm{GHz})$ \\
\hline
\end{tabular}

The temperature sensor was studied in various values of temperature degrees range from $35^{\circ} \mathrm{C}$ (hyperthermia) to $40^{\circ} \mathrm{C}$ (hypothermia) to simulate normal human temperature values. Once a previous parameters setup is complete, then we can use the program to perform numerical simulations. Such simulations are based on solving coupled-mode equations that describe the interaction of guided modes. A grating-assisted device can be designed and analyzed by calculating light propagation, reflection and transmission spectra, the phase group delay, and the dispersion.

To designed the blood pressure sensor, the OptiSystem simulation software (V.16) had been used, the simulation model is shown in figure 2. The OptisystemTM From Optiwave cooperation is a comprehensive software design which permits utilizers for planning and examining optical links within a new networks transmission layer. This tool provides a wide variety of optical and wireless components to plan and implement a complete optical network, which is low cost, time-saving and efficient approach for the researcher. We sought to reach the optimum design for the sensor to read the blood pressure measurement 
of the human body. To execute an effective test of the proposed work we simulated values from (40 diastolic to 190 systolic) $\mathrm{mmHg}$ that represent the limits of human pressure blood values.

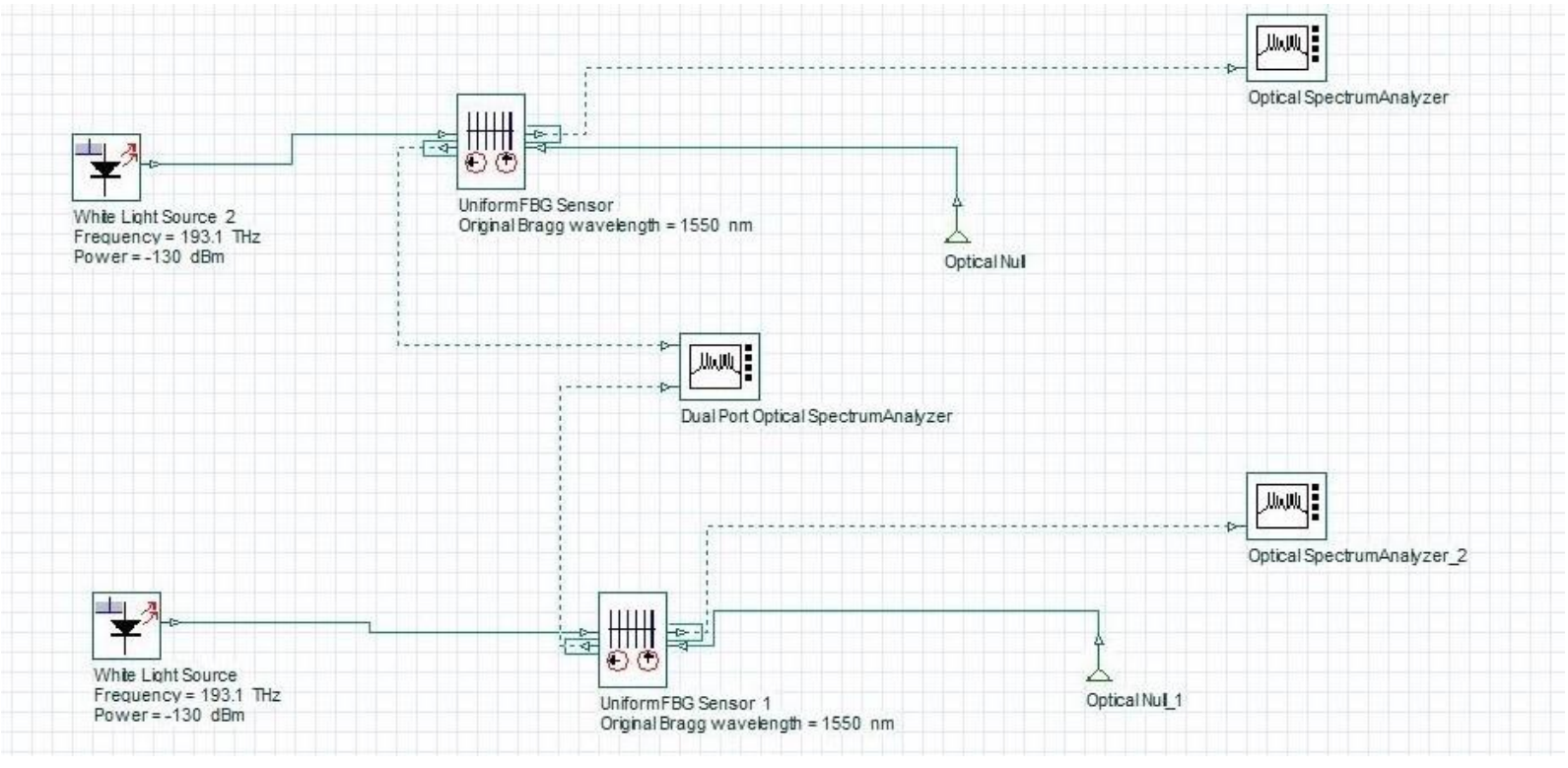

FIG. 2. EXPERIMENTAL SETUP OF ACCURATE FBG PRESSURE SENSOR SYSTEM.

It can be seen that the system generally consists of several parts, i.e., transmitter, FBG and Optical Spectrum Analyzer (OSA). The source was white light source which emits wavelength $1550 \mathrm{~nm}$ and power 0.001 mwatt. This source connection with FBG temperature sensor is having $1550 \mathrm{~nm}$ wavelength and reflectivity 0.9 of the signal passing through. Part of the transmitted signal is reflected (Bragg wavelength), this part was connected to an OSA for monitoring signals after each variation in pressure that contacts the sensing area in FBG.

\section{The Results and Discussions}

The characteristics of FBG have been studied through OptiGrating, the reflection and transmission spectra are shown in figure 3.

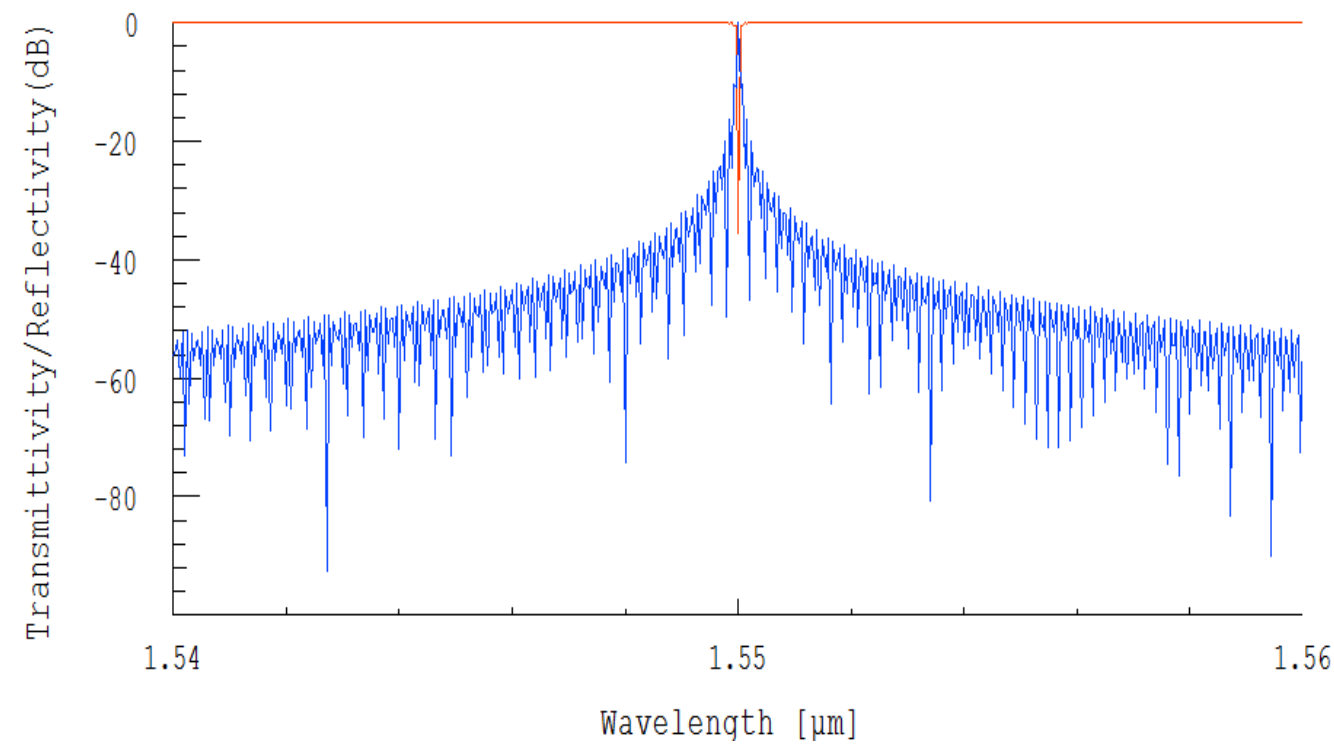

Fig. 3. THE REFLECTION (BLUE) AND TRANSMisSion (RED) SPECTRA OF DESIGNED FBG. 
After taking, analyzing and plotting the values of the data from OptiGrating software, it is observed that by increasing the temperature there is a shift towards longer wavelength (redshift). The obtained spectra illustrate the apparent variance of the change in the Bragg wavelength shift versus the effect of the change in different temperatures as shown in Figure 4. The linear relation between the applied temperature and the shifted Bragg wavelength is illustrated in figure 5 .

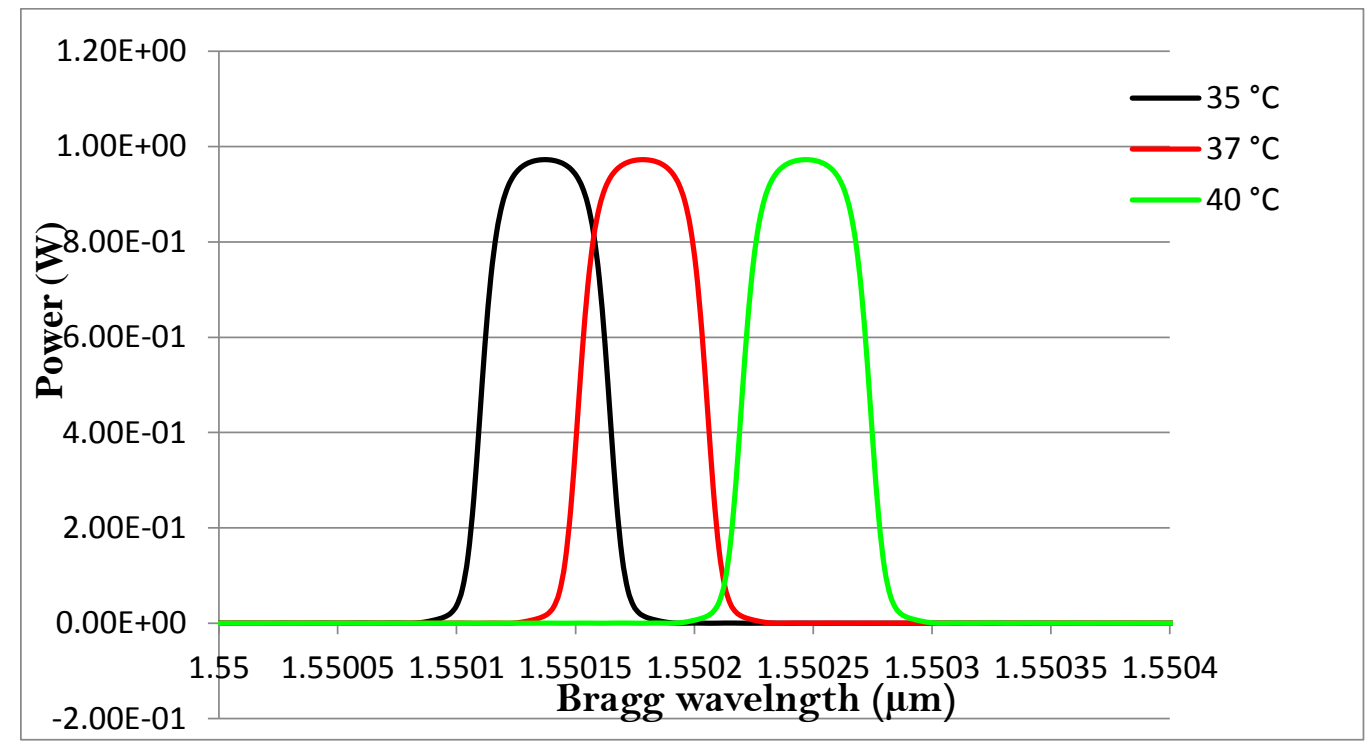

FIG. 4. REFLECTION SPECTRA OF FBG DUE TO APPLIED TEMPERATURE.

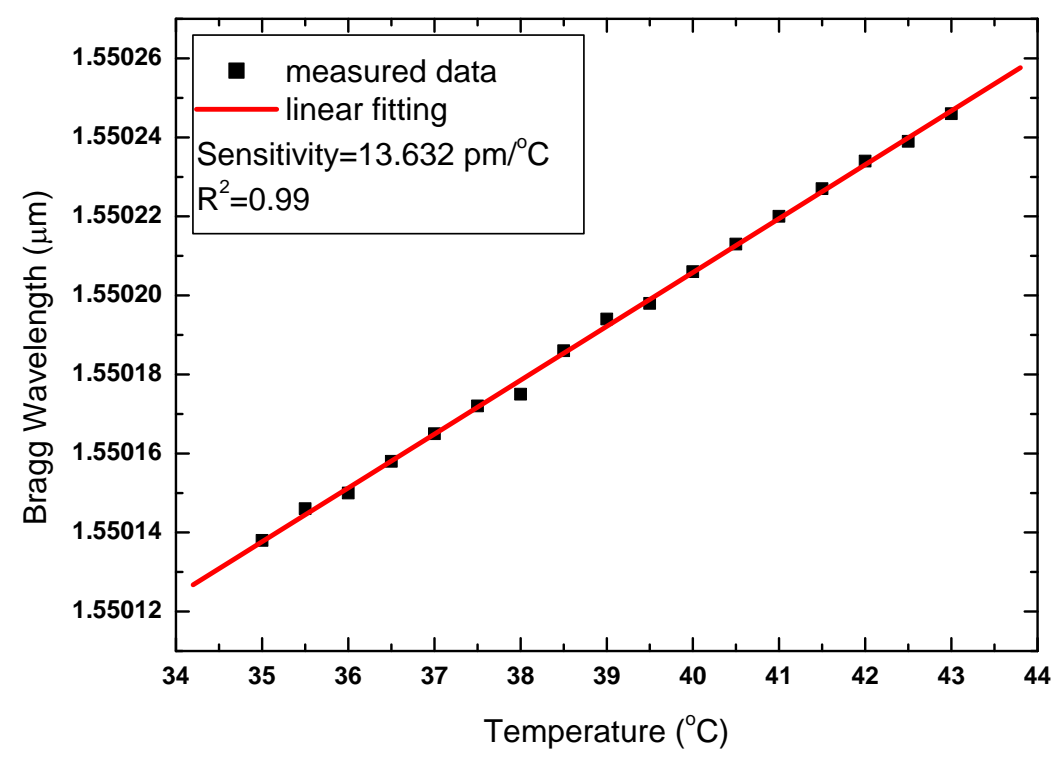

Fig. 5. THE VARIATION OF BRAGG WAVELENGTH DUE TO APPLIED TEMPERATURE.

The sensitivity was calculated to be $\left(13.632 \mathrm{pm} /{ }^{\circ} \mathrm{C}\right)$. The $\mathrm{FBG}$ used $\mathrm{In}$ the current study has been very sensitive to the variation of the temperature degrees, the sensitivity was high when the increasing temperature.

The behaviour of reflected Bragg spectra had been studied due to the applied pressure in the range of human blood pressure, this is shown in figure 6 . 


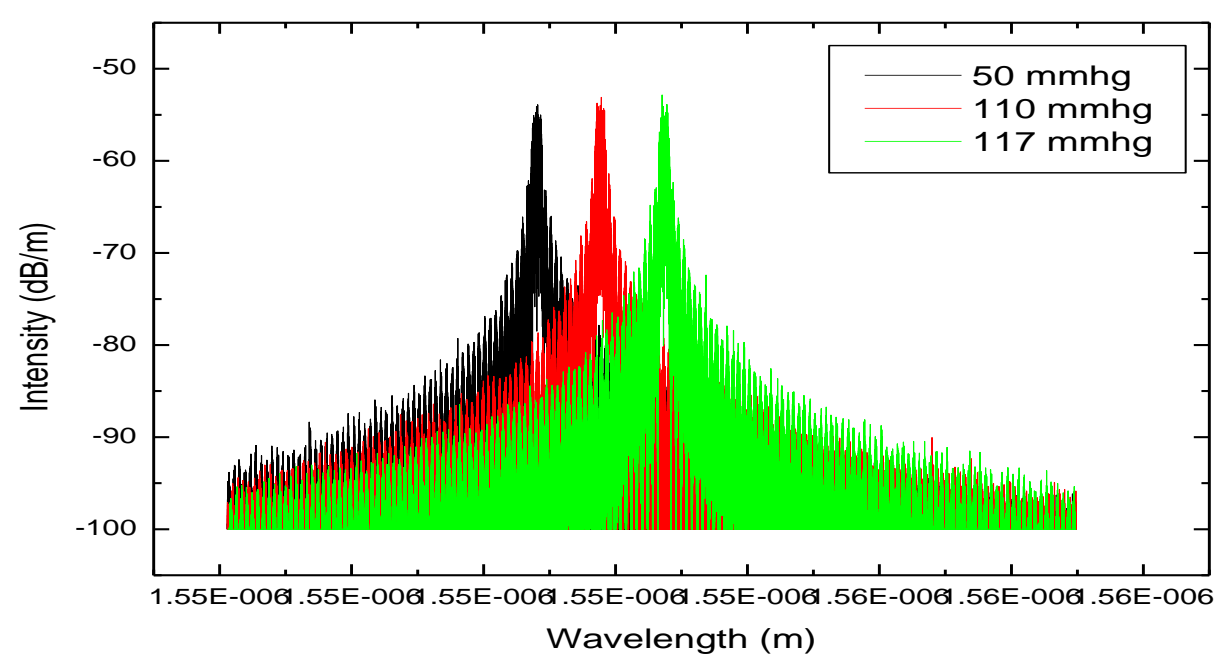

FIG. 6. THE SHIFTED SPECTRA OF REFLECTED BRAGG WAVELENGTH DUE TO APPLIED PRESSURE.

The relation was linear between the various pressure and shifting Bragg wavelength. The sensitivity was calculated to be $(15.75 \mathrm{pm} / \mathrm{mmHg}$ ) as shown in figure 7 .

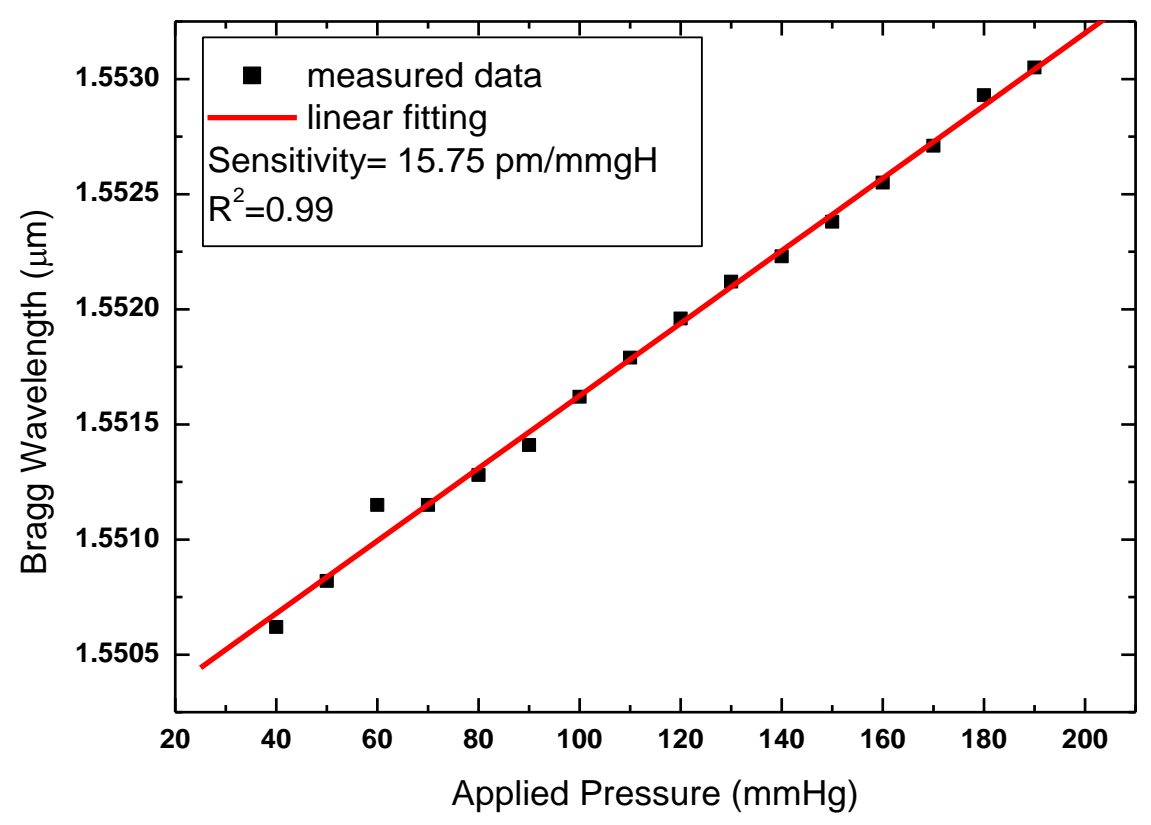

Fig. 7. THE VARIATION OF BRAGG WAVELENGTH DUE TO APPLIED PRESSURE.

From the above results, the submitted design has very good sensitivity to the applied pressure and temperature to detect the vital signs of the human body. The linear relationship of the applied physics and shifting in Bragg wavelength makes it very suitable in medical applications.

\section{Conclusion}

An FBG sensor for detecting temperature and blood pressure for human vital signs has been designed and simulated in this work. it is observed that the relationship between the temperature and Bragg wavelength shifting was linear. this behavior is due to the change of the effective refractive index and the spacing between the gratings $(\Lambda$ due to the applied temperature the main reason for this is the photosensitivity phenomena. And The FBG used 
In the current study has been very sensitive to the variation of the temperature degrees, the sensitivity was $13.632 \mathrm{pm} /{ }^{\circ} \mathrm{C}$ when the increasing temperature. Due to the change in distance between periodic planes created by pressure, the pressure increase causes an increase in Bragg wavelength shift. The output power is a strain-affected variable and has been calculated under similar conditions. The linearity of these graphs proves the FBG hypothesis that the Bragg wavelength change will be increased when the pressure is increased. The sensitivity was calculated to be $\left(15.75 \mathrm{pm} /{ }^{\circ} \mathrm{C}\right)$. The designed sensors were very sensitive to the small variation in applied temperature and pressure, so they could have a very good candidate in medical application. The future work of this technique that we could apply it in sensing technologies and ubiquitous wireless networking is reflected in medical practice, which positively affects the lives of citizens. It provides new healthcare advantages under the scope of e-Health applications.

\section{References}

[1] Wang, Yu, Hongyu Yuan, Xin Liu, Qing Bai, Hongjuan Zhang, Yan Gao, and Baoquan Jin. "A Comprehensive Study of Optical Fiber Acoustic Sensing." IEEE Access 7 (2019): 85821-85837.

[2] Pospíšilová, Marie, Gabriela Kuncová, and Josef Trögl. "Fiber-optic chemical sensors and fiber-optic bio-sensors." Sensors 15, no. 10 (2015): 25208-25259.

[3] Zhang, Y.N.; Zhao, Y.; Zhou, T.M.; Wu, Q.L. "Applications and developments of on-chip biochemical sensors based on optofluidic photonic crystal cavities". Lab Chip 18(2018): 57-74.

[4] Campanella, Carlo Edoardo, Antonello Cuccovillo, Clarissa Campanella, Abdulkadir Yurt, and Vittorio Passaro. "Fibre Bragg grating based strain sensors: review of technology and applications." Sensors 18, no. 9 (2018): 3115.

[5] Petermann, Ingemar. "Fibre Bragg Gratings: Characterization, Realization and Simulation." PhD diss., KTH, 2007.

[6] Kadhim, Shehab A., et al. "Fiber Communication System based on FBG as Dispersion Compensator, Design an Experimental Setup." Journal of Physics: Conference Series. Vol. 1294. No. 2. IOP Publishing, 2019.

[7] Silva, Ricardo M., Hugo Martins, Ivo Nascimento, José M. Baptista, António Lobo Ribeiro, José L. Santos, Pedro Jorge, and Orlando Frazão. "Optical current sensors for high power systems: a review." Applied sciences 2, no. 3 (2012): 602628.

[8] Fukano, Hideki, Daichi Watanabe, and Shuji Taue. "Sensitivity characteristics of multimode-interference optical-fiber temperature-sensor with solid cladding material." IEEE Sensors Journal 16, no. 24 (2016): 8921-8927.

[9] Hao, Y.; Chen, M.Q.; Lv, R.Q.; Xia, F. "In-fiber rectangular air Fabry-Perot strain sensor based on high-precision fiber cutting platform". Opt. Commun. 2017, 384, 107-110.

[10] Chen, Xianfeng, Chi Zhang, David J. Webb, Kyriacos Kalli, and Gang-Ding Peng. "Highly sensitive bend sensor based on Bragg grating in eccentric core polymer fiber." IEEE Photonics Technology Letters 22, no. 11 (2010): 850-852.

[11] Dennison, Christopher Raymond Stuart. "Development and application of in-fibre Bragg grating based biomedical pressure sensors." PhD diss., 2008.

[12] Sethuraman, Gopakumar. "Fiber Bragg Grating (FBG) Based Chemical Sensor." (2008).

[13] Sandra F. H. Correia, Paulo Antunes, Edison Pecoraro, Patrícia P. Lima, Humberto Varum, Luis D. Carlos, Rute A. S. Ferreira and Paulo S. André, "Optical Fiber Relative Humidity Sensor Based on a FBG with a Di-Ureasil Coating", MDBI Sensors, Basel, Switzerland, 2012.

[14] Ibrahim A. Murdas and Ahmed N. Jabbar, "Online Healthcare Monitor System Based on Optical Wireless and FBG Sensors", International Journal of Engineering \& Technology, vol.7 (3.4) P.100-106., 2018.

[15] Yousef S. Kavian and Z. Ghassemlooy, "Intelligent Systems for Optical Networks Design: Advancing Techniques" IGI Global, 2013.

[16] Correia, R., S. James, S. W. Lee, S. P. Morgan, and S. Korposh. "Biomedical application of optical fibre sensors." Journal of Optics 20, no. 7 (2018): 073003.

Received 10 February 2020; Accepted 9 July 2020 
[17] Zhu, Yinian. "Fabrication of long-period gratings and their applications in optical fibre communications and sensing systems." PhD diss., University of Johannesburg, 2002.

[18] M. Nakamura, C. Komatsu, Y. M., K. Fujita, M. Yamauchi, Y. Mizutani, S. Kimura, Y. Suzaki, T. Yokouchi, K. Nakagawa, \& S. Ejima "Evolution of Optical Fiber Bragg Grating Fabrication Using KrF Eximer Laser" Japanese Journal of Applied Physics, Vol.43, No.1, P.P.147-151, 2004.

[19]X. Xu "Improving the Performance of FBG Sensing System" M.Sc. Thesis, School of Electrical, Computer \& Telecommunications, University of Wollongong, 2006.

[20] de Fátima Domingues, Maria, Cátia Tavares, Tiago Leite, Nélia Alberto, Cátia Leitão, Carlos Marques, Ayman Radwan, Eduardo Rocon, Paulo Antunes, and Paulo André. "Fiber Bragg Gratings as e-Health Enablers: An Overview for Gait Analysis Applications." In Applications of Optical Fibers for Sensing. IntechOpen, 2018.

[21] Marcel Fajkus, Jan Nedoma, Radek Martinek,, Alternative fiber optic sensor based on Bragg grating for heart rate monitoring", International Journal of Biosensors \& Bioelectronics,

Volume 4 Issue 5, pp.231-, 2018 .

[22] Bey, Samer K. Abi Kaed, Tong Sun, and Kenneth TV Grattan. "Simultaneous measurement of temperature and strain with long period grating pairs using low resolution detection." Sensors and Actuators A: Physical 144, no. 1 (2008): 83-89.

[23] Rosman, N. A., C. B. M. Rashidi, S. A. Aljunid, and R. Endut. "Temperature monitoring system using fiber Bragg grating (FBG) approach." In AIP Conference Proceedings, vol. 2203, no. 1, p. 020065. AIP Publishing LLC, 2020.

[24] Hutchison, James S., Roxanne E. Ward, Jacques Lacroix, Paul C. Hébert, Marcia A. Barnes, Desmond J. Bohn, Peter B. Dirks et al. "Hypothermia therapy after traumatic brain injury in children." New England Journal of Medicine 358, no. 23 (2008): 2447-2456.

[25] Kario, Kazuomi, Lutgarde Thijs, and Jan A. Staessen. "Blood pressure measurement and treatment decisions: masked and white-coat hypertension." Circulation research 124, no. 7 (2019): 990-1008. 